\title{
Pubertal development and premature ovarian insufficiency in patients with APECED
}

\author{
Viivi Saari ${ }^{1,2}$, Elina Holopainen ${ }^{2,3}$, Outi Mäkitie ${ }^{1,2,4,5}$ and Saila Laakso 1,2,4 \\ ${ }^{1}$ Children's Hospital and Pediatric Research Center, University of Helsinki and Helsinki University Hospital, Helsinki, \\ Finland, ${ }^{2}$ Research Program for Clinical and Molecular Metabolism, Faculty of Medicine, University of Helsinki, \\ Helsinki, Finland, ${ }^{3}$ Department of Obstetrics and Gynecology, University of Helsinki and Helsinki University Hospital, \\ Helsinki, Finland, ${ }^{4}$ Folkhälsan Research Center, Helsinki, Finland, and ${ }^{5}$ Department of Molecular Medicine and \\ Surgery, Karolinska Institutet and Clinical Genetics, Karolinska University Hospital, Stockholm, Sweden
}

Correspondence should be addressed to S Laakso

Email

saila.laakso@helsinki.fi

\begin{abstract}
Objective: To determine the natural course of pubertal development, growth during puberty, and development of POI in females with autoimmune polyendocrinopathy-candidiasis-ectodermal dystrophy (APECED), also called autoimmune polyendocrine syndrome type I.

Design: Longitudinal follow-up study.

Methods: A national cohort of females with APECED aged $\geq 12$ years were followed during 1965-2018. Attainment of adult height was defined when patients' height increased less than $1 \mathrm{~cm}$ per year. Diagnosis of POI was based on delayed puberty or POI symptoms with amenorrhea, and/or FSH $\geq 40 \mathrm{IU} / \mathrm{L}$.

Results: Altogether 40 women with APECED were followed up to the average age of 37.3 (range: 14.6-61.9) years; 16 females (40\%) were $\geq 40$ years. Pubertal development started spontaneously in 34 patients and 29 had spontaneous menarche. POI developed in 28 patients (70\%) at the median age of 16.0 years (range: 11.3-36.5), and in 20 of them (71\%) before attaining adult height. In 11 cases puberty was induced or completed by hormonal therapy. Patients with POI were significantly shorter at menarche, but adult heights did not differ from non-POI females. Patients with POI had more often primary adrenocortical insufficiency ( $93 \%$ vs $58 \%, P=0.017)$ and ovarian antibodies ( $81 \%$ vs $30 \%, P=0.003$ ) compared to those with normal ovarian function $(n=12)$.

Conclusions: POI developed in the majority of patients with APECED, often before or shortly after menarche. Timely commencement of hormonal replacement therapy is important to ensure optimal pubertal development and growth. The possibility of fertility preservation before development of POI in APECED patients should be further studied.
\end{abstract}

\section{Introduction}

Autoimmune

polyendocrinopathy-candidiasisectodermal dystrophy (APECED), also called autoimmune polyendocrine syndrome type I (APS-I), is a rare autosomal recessive disease caused by mutations in the autoimmune regulator (AIRE) gene (21q22.3) (1). APECED usually presents in childhood and adolescence, but the clinical presentation is highly variable (2). Patients have autoantibodies against tissue-specific antigens $(3,4)$. The classic triad of APECED comprises chronic mucocutaneous candidiasis (CMC), hypoparathyroidism
(HP), and primary adrenocortical insufficiency (PAI). Clinical diagnosis of APECED is based on two of the three components of classic triad, or one major manifestation combined with a sibling with APECED (5).

Primary ovarian insufficiency (POI) is the third most common endocrine component of $\operatorname{APECED}(2,5)$. The main symptom of POI is secondary amenorrhea, defined as a lack of regular menstrual cycle for over 4 months. Less frequently ovarian function may wane before completion of pubertal development, causing primary

Published by Bioscientifica Ltd. 
amenorrhea (6). Diagnosis is confirmed by detection of raised FSH and declined estradiol concentrations in the serum (7). FSH concentration $\geq 40 \mathrm{IU} / \mathrm{L}$ in women under the age of 40 years is considered a diagnostic criterion of POI (8). Ovarian function may fluctuate in up to $50 \%$ of the patients with POI. Approximately $5-10 \%$ of women conceive spontaneously and deliver a child after POI diagnosis (7). Pregnancies with donated ovum or embryo are possible in patients with POI (9).

POI may have significant health implications. Hypoestrogenism may lead to vasomotor symptoms (hot flashes and night sweats) and sleep disturbance $(10,11)$. POI has been associated with increased overall mortality, cardiovascular complications, neurological and psychiatric diseases, and osteoporosis. The risk of adverse outcomes increases with earlier age at the time of POI diagnosis (8). Long-term hormone replacement therapy (HRT) is needed for relief of menopausal symptoms and to prevent long-term health consequences of estrogen deficiency (9).

The prevalence of POI has been described in some cohorts of patients with APECED to vary between 33 and $69 \%(5,12,13,14)$. However, no studies have systematically explored natural course of POI and how POI affects pubertal development, growth, gynecologic health and fertility in patients with APECED. Here we provide a longitudinal analysis on ovarian function in 40 women with APECED using data from the large Finnish cohort with unique follow-up time of up to over 50 years. The study focuses especially on onset and progression of puberty and growth, diagnosis and symptoms of POI, and fertility. Furthermore, since estrogen status has been linked to decreased expression of AIRE and increased appearance of autoimmune manifestations (15), we also analyzed the sequence of new endocrinopathies in relation to pubertal phase and different stages of ovarian function. Our findings indicate that POI develops in the majority of females with APECED already during puberty or shortly after reaching adult height and impacts pubertal growth but not final height.

\section{Subjects and methods}

\section{Patients}

All female patients with APECED $(n=48)$ were identified from the previously described national cohort of Finnish patients (5). The cohort has later been complemented by continuous recruitment of subsequently diagnosed patients from tertiary pediatric and adult endocrine centers throughout the country (16). Eight patients (17\%) were excluded from the analysis due to lack of patient consent, lack of adequate data on ovarian function, or age below 12 years at the end of follow-up when POI diagnosis could not yet be set. Consequently, 40 Finnish female patients with APECED were included in the study. Flow chart of patient selection is presented in Fig. 1. An ethical approval was obtained from the Research Ethics Committee of the Hospital District of Helsinki and Uusimaa. Informed written consent was obtained from all study participants or their guardians (subjects aged $<18$ years).

\section{Clinical data and diagnostic criteria for POI}

Medical records were collected from all identified health service providers from 1965 to the end of 2018 and reviewed for clinical data, growth, and biochemical parameters. Age at diagnosis was determined for the following disease manifestations: CMC, PAI, HP, POI, hypothyroidism, growth hormone deficiency, diabetes,

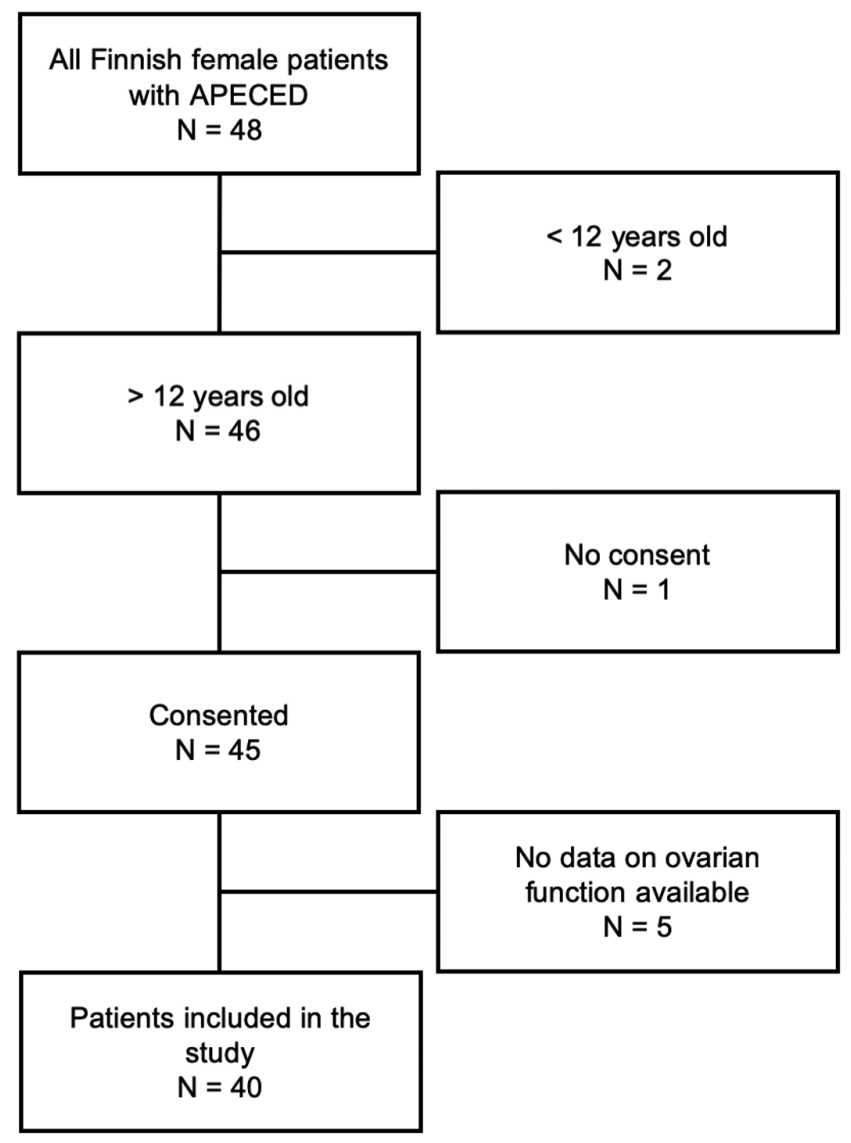

Figure 1

Flowchart of patient selection in the study. 
hepatitis, tubulointerstitial nephritis, keratitis, alopecia, and vitiligo. Pubertal development was classified according to Tanner scale (17) based on clinical evaluation by the attending pediatric endocrinologist responsible for the patient's care. Height and BMI SD-scores were calculated according to Finnish growth references (18). Attainment of adult height was defined when longitudinal growth decelerated, and patients' height increased less than $1 \mathrm{~cm}$ per year. Serum FSH values, obtained before HRT onset or after adequate wash out period of HRT, were available for 30 patients $(75 \%)$.

Diagnosis of POI was based on delayed puberty (patient had not reached stage 2 of breast development by the age of 13 years or menarche by the age of 15 years) or POI symptoms with amenorrhea, and/or FSH $\geq 40$ IU/L under the age of 40 years. The ovarian antibodies were analyzed in a routine diagnostic laboratory with an indirect immunofluorescence assay using standard techniques between 1982 and 2016 .

\section{Statistical analysis}

Results are reported as median (range) or mean (95\% CI). Fisher's exact test was used for categorical variables and Mann-Whitney $U$-test or independent samples t-test for continuous variables. A $P$ value below 0.05 was considered to indicate statistical significance. IBM SPSS statistics version 25 was used for statistical analyses.

\section{Results}

\section{Patient characteristics}

The study cohort included altogether 40 female patients with APECED who were followed until the age of 14.6-61.9 years (median 37.7 years). Patients were followed clinically for 1-12 times yearly by a pediatric endocrinologist during pubertal development, depending on the severity of disease, and usually yearly thereafter. Demographics of the patients are presented in Table 1. The first disease manifestation occurred at the median age of 4.2 years, and APECED diagnosis was set at the median age of 6.9 years. In ten patients (25\%), APECED was diagnosed only after the onset of puberty. At the end of follow-up, 16 patients (40\%) had reached the age of 40 years, the upper age limit for POI diagnosis. Thirty-one patients $(78 \%)$ were alive, and nine (23\%) patients had deceased during follow-up at 19-55 years of age. Causes of death in the nine deceased patients were endocrine and
Table 1 Demographics of the 40 female patients with autoimmune polyendocrinopathy-candidiasis-ectodermal dystrophy (APECED).

\begin{tabular}{l} 
Characteristics \\
\hline Age at onset of first disease \\
manifestation (years) \\
Age at diagnosis of APECED (years) \\
APECED-diagnosis in prepuberty \\
Age at last follow-up (years) \\
Age at death ( $n=9$, years) \\
AIRE genotype \\
C.769C $>$ T/c.769C $>$ T \\
c.769C $>$ T/other \\
c.769C $>$ T/x \\
Unknown*
\end{tabular}

\begin{tabular}{c} 
Median (range) or $n(\%)$ \\
\hline $4.2(0.1-14.7)$ \\
$6.9(0.6-25.0)$ \\
$30(75 \%)$ \\
$37.3(14.6-61.9)$ \\
$36(19-55)$ \\
$30(75 \%)$ \\
$6(16 \%)$ \\
$2(5 \%)$ \\
$2(5 \%)$
\end{tabular}

*One deceased patient.

$A I R E$, autoimmune regulator gene.

metabolic diseases $(n=2)$, alcohol related $(n=2)$, oral and esophageal neoplasms $(n=1)$, diseases of the circulatory systems $(n=1)$, neurological $(n=1)$, and unknown $(n=2)$.

Thirty patients (75\%) were homozygous for the Finnish major mutation on AIRE c.769C $>\mathrm{T}$ (p.Arg257Ter), while in the others it was compounded with c.967_979del13 (p.Leu323fs) $(n=2), \quad$ c.932G $>$ A (p.Cys311Tyr) $(n=2)$, c.1638A $>$ T (p.Ter546Cys) $(n=1)$ or c.1163^1164insA (p.Met388fs) $(n=1)$. Only one c.769C $>$ T variant was found in two patients, and AIRE genotype was not defined in two patients (Table 1); all these four females fulfilled the clinical diagnostic criteria for APECED.

\section{Pubertal development and diagnosis of POI}

Median age at the beginning of spontaneous stage 2 of breast development was 12.2 years in 34 patients (range, 10.2-16.2), and the median age at spontaneous menarche was 13.8 years in 29 patients (12.0-19.5). POI was diagnosed in 28 of the 40 women with APECED (70\%). Diagnostic data for POI is summarized in Table 2. In 11 cases, POI developed already before menarche reflecting early-onset ovarian insufficiency. HRT was started to them to complete pubertal development at the median age of 14.7 (range, 11.3-22.0). In 17 patients with POI (61\%), POI was diagnosed after menarche leading to secondary amenorrhea. FSH concentration was $\geq 40 \mathrm{IU} / \mathrm{L}$ in $17 / 23$ (74\%), and ovarian antibodies were present in 21/27 (78\%) of patients with POI. In four patients with POI, ovarian antibodies fluctuated, becoming negative at the median age of 40 years (range, 31.8-46.5). Six of 16 over 40-yearold patients did not developed POI; four had experienced 
Table 2 Clinical characteristics of 28 patients with autoimmune polyendocrinopathy-candidiasis-ectodermal dystrophy (APECED) at the time of primary ovarian insufficiency (POI) diagnosis.

\begin{tabular}{|c|c|c|c|}
\hline Characteristics & $n / m e d i a n$ & $\%$ & Range \\
\hline Age at POI diagnosis (years) & 16.0 & & $11.3-36.5$ \\
\hline POI before B2 & 6 & 21 & \\
\hline $\begin{array}{l}\text { POI between } B 2 \text { and } \\
\text { menarche }\end{array}$ & 5 & 18 & \\
\hline $\begin{array}{l}\text { POI between menarche and } \\
\text { age of } 40\end{array}$ & 17 & 61 & \\
\hline POI symptoms & 26 & 93 & \\
\hline Age (years) & 15.5 & & $12.6-31.1$ \\
\hline Symptoms before B2 & 6 & 23 & \\
\hline $\begin{array}{l}\text { Symptoms between B2 and } \\
\text { menarche }\end{array}$ & 4 & 15 & \\
\hline $\begin{array}{l}\text { Symptoms between menarce } \\
\text { and age of } 40\end{array}$ & 16 & 62 & \\
\hline $\mathrm{FSH} \geq 40 \mathrm{IU} / \mathrm{L}^{*}$ & 17 & 74 & \\
\hline Age (years) & 15.7 & & $11.3-39.8$ \\
\hline$\geq 40$ before $B 2$ & 2 & 12 & \\
\hline $\begin{array}{l}\geq 40 \text { between } \mathrm{B} 2 \text { and } \\
\text { menarche }\end{array}$ & 2 & 12 & \\
\hline $\begin{array}{l}\geq 40 \text { between menarche and } \\
\text { age of } 40\end{array}$ & 13 & 76 & \\
\hline Ovarian antibodies positive ${ }^{\dagger}$ & 21 & 78 & \\
\hline Age (years) & 16.8 & & $8.5-39.1$ \\
\hline Positive before B2 & 5 & 24 & \\
\hline $\begin{array}{l}\text { Positive between B2 and } \\
\text { menarche }\end{array}$ & 4 & 19 & \\
\hline $\begin{array}{l}\text { Positive between menarche } \\
\text { and age of } 40\end{array}$ & 12 & 57 & \\
\hline
\end{tabular}

* Measured in 23 (82\%) subjects; ${ }^{\dagger}$ Measured in 27 (96\%) subjects. B2, stage 2 of breast development.

natural menopause at the age of 47.0-57.3 years and two were premenopausal at 40.7 and 42.7 years of age.

\section{Effects of POI on growth during puberty}

In order to examine how POI affected patients' growth during puberty, patients were divided into two groups: (i) POI diagnosed before adult height $(n=20)$ and (ii) POI developed after adult height was reached and patients with normal ovarian function $(n=19)$. Adult height was considered to be reached when longitudinal growth decelerated and patients' height increased less than $1 \mathrm{~cm}$ per year. Adult height was reached at the median age of 17.0 years (range: 15.3-24.0). The youngest patient (14.6 years) had not reached adult height based on growth data. Changes in height and BMI Z-scores during puberty are illustrated in Fig. 2. Patients who developed POI during pubertal growth were, at menarche, shorter than patients with normal ovarian growth. However, with adequate hormonal replacement therapy their growth improved and the differences between groups disappeared. Adult heights did not differ between the groups (height SDS, group 1 vs group 2 , mean (95\% CI), -1.30 (-1.82 to 0.77 ) vs $-0.95(-1.53$ to 0.38$), P=0.52)$. Similarly, no difference was observed between the groups in BMI SDS at the onset of puberty or at time of menarche (Fig. 2). Overall the 39 adult female patients with APECED were shorter [median height SDS (range): $-1.30(-4.94$ to +1.25$)$ ] and more slender than average female Finns [BMI SDS: -0.58 $(-2.34$ to +1.29$)]$.

\section{Comparison between patients with and without POI}

Pubertal timing, defined by age at stage 2 of breast development or at menarche, did not significantly differ between patients with POI $(n=28)$ and patients with normal ovarian function $(n=12$, Table 3$)$. However, hormonal treatment was needed in 11 cases to complete pubertal development. Patients with POI had PAI more often than patients without POI (93\% vs 58\%). Most commonly POI followed PAI $(n=24)$, but in two patients, PAI was diagnosed only many years after POI. Ovarian antibodies were positive in $81 \%$ of patients with POI. However, positive ovarian antibodies were found also in 3 out of 10 (30\%) patients without POI; these antibodypositive patients were $19.3,40.7$, and 54.5 years at the end of follow-up.

Eleven out of the 40 female patients (25\% of POI patients and 33\% of patients without POI) had been pregnant. Pregnancy history of patients with APECED is presented in Table 4. There was no significant difference in the number of spontaneous pregnancies or deliveries between patients with POI and patients with regular ovarian function.

\section{Clinical course of APECED in relation to pubertal status and estrogen status}

In order to determine how estrogen status affects appearance of autoimmune manifestations, we analyzed in 40 patients with APECED, the sequence of new clinical manifestations in relation to pubertal stage and different stages of ovarian function: prepuberty, early puberty (stage 2 of breast development - menarche), late puberty (menarche - adult height), fertile age (adult height - menopause/discontinuation of HRT), and after menopause (Fig. 3). In prepuberty, 18 (45\%) of the patients presented all three manifestations of the classical triad; HP and CMC being the two most common 
$\rightarrow$ No POI before adult height

$\rightarrow$ POI before adult height
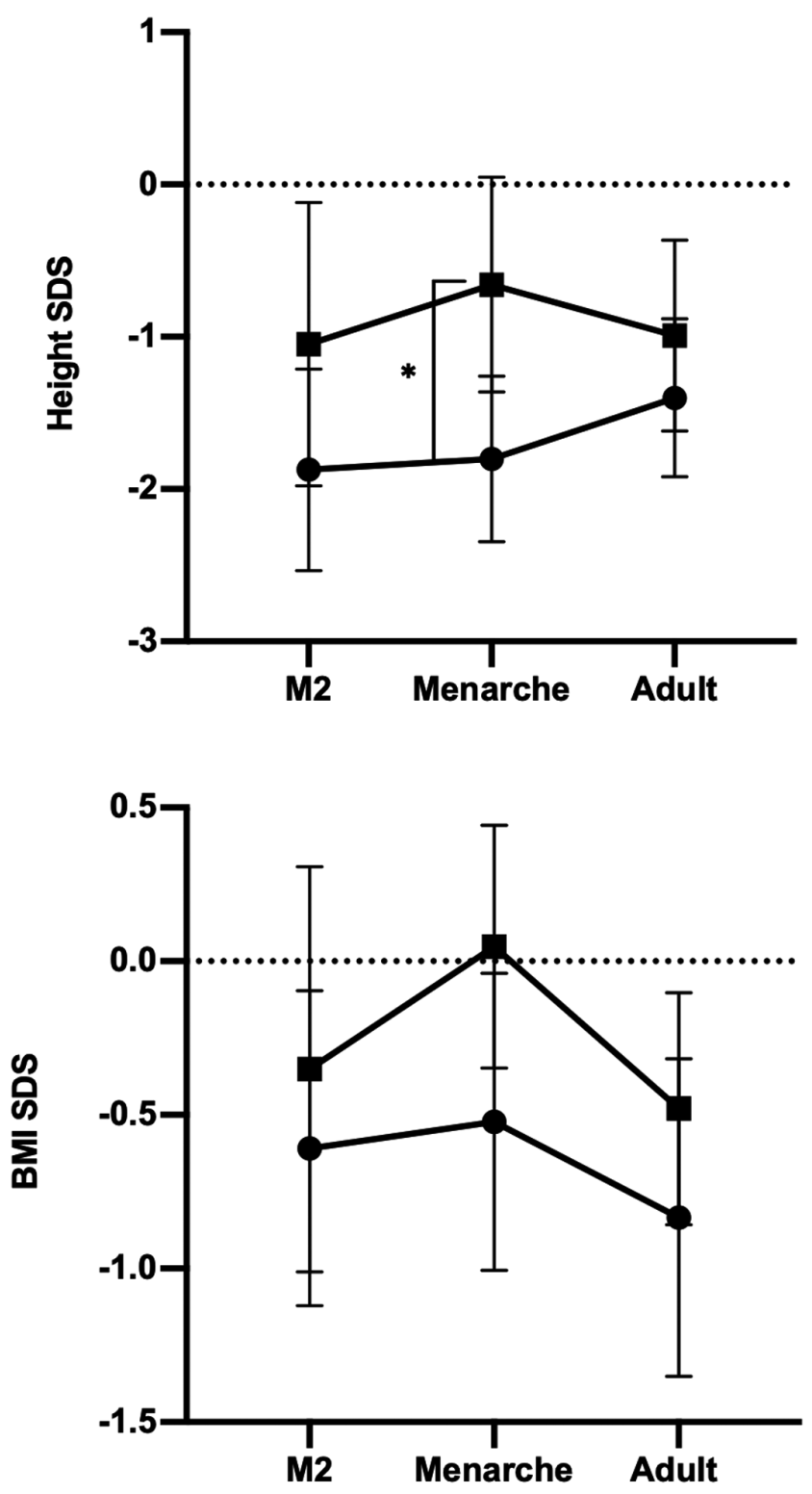

Figure 2

Comparison of mean height and BMI SD-scores (95\% Cl) during different stages of puberty in 20 patients with APECED who developed primary ovarian insufficiency (POI) before adult height attainment and 19 patients with normal ovarian function before adult height attainment. $* P<0.05$.

manifestations diagnosed in 88 and $80 \%$ of the patients. PAI was diagnosed prepubertally in 21 (53\%) and after the onset of puberty in 12 (30\%) patients. POI was diagnosed most often after the onset of puberty but before reaching of adult height. Hypothyroidism, diabetes mellitus, keratoconjunctivitis, and tubulointerstitial nephritis were the only manifestations that occurred more commonly after adult height attainment and only rarely before that. Growth hormone deficiency was diagnosed in five patients: in two patients it was diagnosed before the stage 2 of breast development (B2), in one patient between B2 and menarche, and in two patients after menarche but before reaching adult height.

\section{Discussion}

We analyzed pubertal development and POI in a unique cohort of 40 female patients followed longitudinally for up to over 50 years for APECED. POI was diagnosed in $70 \%$ of the patients. Symptoms and timing of POI were variable but most commonly POI developed shortly after menarche. Adult height and parity were equally compromised in patients with or without POI.

Significantly lower prevalence of POI has been illustrated in three previous large APECED cohorts. In a Russian study involving 33 females with APECED, POI was diagnosed in $48 \%$ (12). In a Norwegian study of 24 females POI was diagnosed in 33\% (13), and in a study carried out in the United States the prevalence was 38\% in 21 female patients (14). In those studies, diagnostic criteria of POI were not described $(13,14)$ or POI was diagnosed also after the age of 40 years (12). Consequently, differences in prevalence of POI among women with APECED can be explained by different diagnostic criteria of POI. In those previous studies, age at the onset of POI varied between the median age of 15-18 $(12,13,14)$. In our series diagnosis was set in $71 \%$ of patients already before they had reached adult height, and most commonly shortly after menarche. Patients had spontaneous menarche at the median age of 13.8 (range, 12.0-19.5), which is slightly later than in the general Finnish population (mean $13.2 \pm 0.02$ years) (19). Primary amenorrhea was diagnosed in 11 (28\%) of the females with APECED, whereas the incidence of primary amenorrhea is $0.9 \%$ in Finnish girls (19). BMI did not significantly differ at stage 2 of breast development or menarche between the groups with or without POI. Thus, malnutrition or high doses of glucocorticoid replacement treatment did not explain slow puberty or amenorrhea. POI developed often during pubertal development, before completion of spontaneously started puberty. Consequently, for diagnosing potential arrested pubertal development, careful and frequent follow-up are warranted through pubertal years. 
Table 3 Comparison of timing of autoimmune polyendocrinopathy-candidiasis-ectodermal dystrophy (APECED) diagnosis, clinical phenotype of the disease, pubertal development, and ovarian antibodies between APECED patients with ( $n=28)$ and without $(n=12)$ primary ovarian insufficiency (POI). Data are presented as median (range) or as $n(\%)$.

Characteristics

Age at APECED diagnosis (years)

Age at last follow-up (years)

Number of manifestations at menarche $(n)$

Chronic mucocutaeous candidiasis (CMC)

Age at CMC diagnosis

Hypoparathyroidism

Primary adrenal insufficiency (PAI)

Age at PAl diagnosis

Age at B2 (years)

Age at menarche (years)

Positive ovarian antibodies*

\begin{tabular}{c} 
POI $(n=28)$ \\
\hline $6.6(2.3-25)$ \\
$35.9(15.8-61.9)$ \\
$4(1-8)$ \\
$27(96 \%)$ \\
$5.9(0.1-25.1)$ \\
$28(100 \%)$ \\
$26(93 \%)$ \\
$10.3(2.5-32.9)$ \\
$12.7(10.5-22.6)$ \\
$14.0(12.0-22.6)$ \\
$22 / 27(81 \%)$
\end{tabular}

\begin{tabular}{c}
\hline Non-POI $(n=12)$ \\
\hline $12.5(0.6-25)$ \\
$40.2(14.6-60.0)$ \\
$3(2-6)$ \\
$12(100 \%)$ \\
$5.6(0.2-23.3)$ \\
$11(92 \%)$ \\
$7(58 \%)$ \\
$9.6(5.5-30.4)$ \\
$11.6(10.2-14.7)$ \\
$14.0(12-19.5)$ \\
$3 / 10(30 \%)$ \\
\hline
\end{tabular}

\begin{tabular}{c} 
P-value \\
\hline 0.41 \\
0.87 \\
0.096 \\
$>0.99$ \\
0.59 \\
0.30 \\
$\mathbf{0 . 0 1 7}$ \\
0.75 \\
0.49 \\
0.78 \\
$\mathbf{0 . 0 0 3}$ \\
\hline
\end{tabular}

*Taken from 37 patients, $n(\mathrm{POI})=27$.

B2, stage 2 of breast development. Bold values were considered statistically significant $(P<0.05)$.

Adult height was decreased in the whole cohort but was equal in patients with or without POI. Patients with POI had delayed longitudinal growth at menarche, but during late puberty they reached similar adult height as the patients with normal ovarian function. This presumably reflected the effects of correctly timed and slowly increased doses of estrogen substitution allowing slow bone maturation while inhibiting too early epiphyseal ossification. Adequate and in time started HRT are important to ensure optimal longitudinal growth, maximal peak bone mass, development of secondary sexual characteristics, and growth of uterus. Adequate HRT is also important for psychosocial well-being. In addition to HRT for POI, the adequate treatment of other manifestations is needed to ensure the optimal longitudinal growth. For example, growth hormone deficiency can disturb normal longitudinal growth and may develop during adolescence.
Pregnancies were uncommon as only 11 (27\%) of the 40 women with APECED had been pregnant. We found in all 15 recorded pregnancies, three (20\%) ending up to a miscarriage. A Russian study has previously reported seven pregnancies amongst 33 females with APECED, but miscarriage rate was not reported (12). We could not study the reasons behind diminished fertility in this retrospective analysis. It has been suggested that in addition to POI, other endocrinopathies or immunological defects may have negative effects on the prognosis of pregnancy (20). Furthermore, recent mice studies have indicated that defects in AIRE can lead to disturbed early embryonic development and implantation (21). These effects of AIRE on fertility should be further explored and may be important factors also in pregnancies with ovum donation. Early progression of POI before or right after menarche complicates the management of fertility for these women since ovum freezing, in general, is clinical practice only after puberty. The possibility and effectiveness of fertility

Table 4 Clinical characteristics of 15 pregnancies in 11 patients from the total cohort of 40 APECED patients with and without primary ovarian insufficiency (POI).

\begin{tabular}{l} 
Characteristics \\
\hline Ever been pregnant \\
Number of pregnancies \\
Number of spontaneous pregnancies \\
Number of pregnancies via infertility treatments \\
Deliveries \\
From spontaneous pregnancies \\
Spontaneous abortion \\
From spontaneous pregnancies \\
Induced abortions \\
From spontaneous pregnancies
\end{tabular}

\begin{tabular}{c}
\hline POI $(n=28)$ \\
\hline $7(25 \%)$ \\
8 \\
4 \\
$4^{*}$ \\
4 \\
2 \\
3 \\
2 \\
1 \\
1 \\
\hline
\end{tabular}

\begin{tabular}{c}
\hline Non-POI $(n=12)$ \\
\hline $4(33 \%)$ \\
7 \\
5 \\
$2^{\dagger}$ \\
5 \\
3 \\
- \\
- \\
2 \\
2 \\
\hline
\end{tabular}

\begin{tabular}{c}
\hline P-value \\
\hline 0.68 \\
0.32 \\
0.13 \\
$>0.99$ \\
0.86 \\
$>0.99$ \\
- \\
- \\
$>0.99$ \\
$>0.99$ \\
\hline
\end{tabular}

*All via ovum donation; ${ }^{\dagger}$ All with own ovum. 


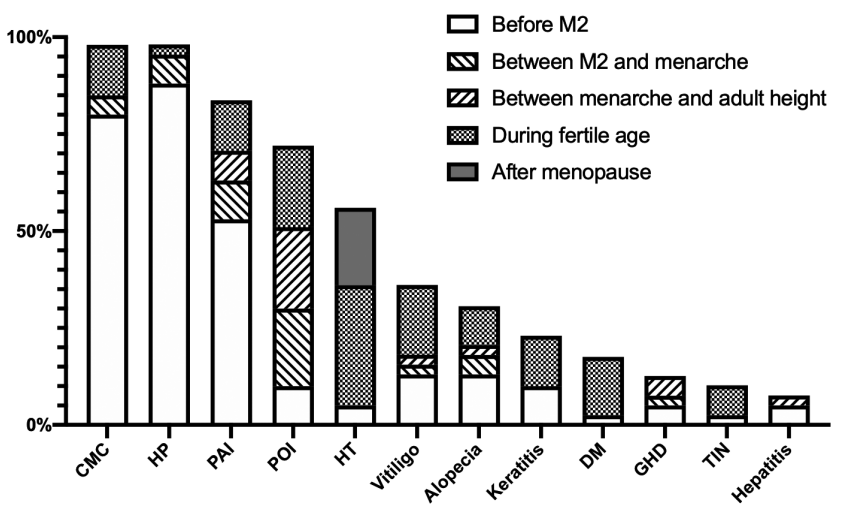

Figure 3

Appearance of clinical manifestations in 40 female patients with APECED in relation to pubertal stage and menopause. CMC, chronic mucocutaneous candidiasis; HP, hypoparathyroidism; PAl, primary adrenocortical insufficiency; POI, primary ovarian insufficiency; $\mathrm{HT}$, hypothyroidism; DM, diabetes mellitus; GHD, growth hormone deficiency; TIN, tubulointerstitial nephritis.

preservation with ovarian stimulation and ovum freezing before development of POI in APECED patients should be further studied. Currently, no immunomodulatory medication or other treatment options are available to prevent appearance of new manifestations including POI in patients with APECED.

The clinical course of APECED is highly variable, but the effects of ovarian function on the clinical phenotype have not been explored before. It has been suggested that the level of AIRE is linked to sexual dimorphism of susceptibility to autoimmune diseases - females being more susceptible to these diseases than males. In human and mouse thymus, females express less AIRE than males. Moreover, estrogen treatment results in down regulation of AIRE expression in cultured human thymic epithelial cells (15). In addition, a recent study on infant human thymic tissue showed differences in AIRE-interaction networks between genders during and after minipuberty (22). Here we were able to describe in a longitudinally followed cohort how defects on AIRE caused different manifestations during different stages of puberty and ovarian function. Manifestations of the classical triad (CMC, HP, PAI) presented often prepubertally. Hypothyroidism and diabetes mellitus appeared mainly postpubertally, after adult height had been reached and even after the age of menopause. Overall, the clinical phenotype associated with stages of ovarian function and the underlying, still unknown mechanisms warrant further studies.
Altogether nine patients in our cohort had deceased between 19 and 55 years of age. We and other have previously shown high mortality in patients with APECED $(12,13)$. Causes of death in our patients were in line with a recent study reporting increased mortality among the Finnish patients with APECED (23).

Our study was limited by the small number of included patients. It is still noteworthy that this is the largest study to date and the follow-up of these patients were thorough. The retrospective nature of the study also introduces potential limitations. For example, we were not able to use side-chain cleavage enzyme (SCC)-antibody results in our analysis because they were measured only in a small number of patients even though they are presently considered better predictors of ovarian insufficiency than ovarian antibodies $(5,11,24$, 25). During follow-up time of over 50 years, diagnostic methods and clinical practice in pubertal induction and treatment of POI may have changed. However, we were able to represent the whole potentially fertile time period especially in 16 patients that reached the age of 40 years during the follow-up time.

We conclude that since POI may develop already before onset of spontaneous pubertal development or before completion of spontaneously started development and adult height, careful and frequent follow-up are warranted in all females with APECED. Timely commencement of hormonal replacement therapy is important to ensure similar pubertal timing with peers, normal growth, and normal bone density. The effect of APECED and POI in fertility and prognosis of pregnancies in women with APECED warrant further studies.

\section{Declaration of interest}

The authors declare that there is no conflict of interests that could be perceived as prejudicing the impartiality of this study.

\section{Funding}

This work was supported by grants from Pediatric Research Center, Helsinki University Hospital (S L); Helsinki University Hospital (O M, E H); The Finnish Foundation for Pediatric Research (S L); The Finnish Medical Foundation (S $\mathrm{L})$; Academy of Finland (O M); Sigrid Jusélius Foundation (O M); Folkhälsan Research Foundation (O M); Novo Nordisk Foundation (O M).

\section{Data availability}

Restrictions apply to the availability of data generated or analyzed during this study to preserve patient confidentiality. The corresponding author will on request detail the restrictions and any conditions under which access to some data may be provided. 


\section{Acknowledgements}

The authors would like to acknowledge the work of research nurse Nea Boman.

\section{References}

1 Aaltonen J, Björses P, Sandkuijl L, Perheentupa J \& Peltonen L. An autosomal locus causing autoimmune disease: autoimmune polyglandular disease type I assigned to chromosome 21. Nature Genetics 19948 83-87. (https://doi.org/10.1038/ng0994-83)

2 Ahonen P, Myllärniemi S, Sipilä I \& Perheentupa J. Clinical variation of autoimmune polyendocrinopathy-candidiasis-ectodermal dystrophy (APECED) in a series of 68 patients. New England Journal of Medicine 1990322 1829-1836. (https://doi.org/10.1056/ NEJM199006283222601)

3 Meloni A, Furcas M, Cetani F, Marcocci C, Falorni A, Perniola R, Pura M, Bøe Wolff AS, Husebye ES, Lilic D et al. Autoantibodies against Type I interferons as an additional diagnostic criterion for autoimmune polyendocrine syndrome Type I. Journal of Clinical Endocrinology and Metabolism 200893 4389-4397. (https://doi. org/10.1210/jc.2008-0935)

4 Meager A, Visvalingam K, Peterson P, Möll K, Murumägi A, Krohn K, Eskelin P, Perheentupa J, Husebye E, Kadota Y et al. Anti-interferon autoantibodies in autoimmune polyendocrinopathy syndrome Type 1. PLoS Medicine 20063 e289. (https://doi.org/10.1371/journal. pmed.0030289)

5 Perheentupa J. Autoimmune polyendocrinopathy-candidiasisectodermal dystrophy. Journal of Clinical Endocrinology and Metabolism 200691 2843-2850. (https://doi.org/10.1210/jc.2005-2611)

6 Rebar RW \& Connolly HV. Clinical features of young women with hypergonadotropic amenorrhea. Fertility and Sterility $1990 \mathbf{5 3}$ 804-810. (https://doi.org/10.1016/S0015-0282(16)53513-4)

7 Nelson LM. Clinical practice. Primary ovarian insufficiency. New England Journal of Medicine 2009360 606-614. (https://doi. org/10.1056/NEJMcp0808697)

8 De Vos M, Devroey P \& Fauser BC. Primary ovarian insufficiency. Lancet 2010376 911-921. (https://doi.org/10.1016/S01406736(10)60355-8)

9 Goswami D \& Conway GS. Premature ovarian failure. Human Reproduction Update 200511 391-410. (https://doi.org/10.1093/ humupd/dmi012)

10 Davis SR. Premature ovarian failure. Maturitas 199623 1-8. (https:// doi.org/10.1016/0378-5122(95)00966-3)

11 Yeganeh L, Boyle JA, Wood A, Teede H \& Vincent AJ. Menopause guideline appraisal and algorithm development for premature ovarian insufficiency. Maturitas 2019130 21-31. (https://doi. org/10.1016/j.maturitas.2019.09.009)

12 Orlova EM, Sozaeva LS, Kareva MA, Oftedal BE, Wolff ASB, Breivik L, Zakharova EY, Ivanova ON, Kämpe O, Dedov II et al. Expanding the phenotypic and genotypic landscape of autoimmune polyendocrine syndrome Type 1. Journal of Clinical Endocrinology and Metabolism 2017102 3546-3556. (https://doi.org/10.1210/ jc.2017-00139)

13 Bruserud $\varnothing$, Oftedal BE, Landegren N, Erichsen MM, Bratland E, Lima K, Jørgensen AP, Myhre AG, Svartberg J, Fougner KJ et al. A longitudinal follow-up of autoimmune polyendocrine syndrome Type 1. Journal of Clinical Endocrinology and Metabolism 2016101 2975-2983. (https://doi.org/10.1210/jc.2016-1821)

14 Ferre EMN, Rose SR, Rosenzweig SD, Burbelo PD, Romito KR, Niemela JE, Rosen LB, Break TJ, Gu W, Hunsberger S et al. Redefined clinical features and diagnostic criteria in autoimmune polyendocrinopathy-candidiasis-ectodermal dystrophy. JCI Insight 20161 e88782. (https://doi.org/10.1172/jci.insight.88782)

15 Dragin N, Bismuth J, Cizeron-Clairac G, Biferi MG, Berthault C, Serraf A, Nottin R, Klatzmann D, Cumano A, Barkats M et al. Estrogen-mediated downregulation of AIRE influences sexual dimorphism in autoimmune diseases. Journal of Clinical Investigation 2016126 1525-1537. (https://doi.org/10.1172/JCI81894)

16 Laakso S, Borchers J, Toiviainen-Salo S, Pekkinen M \& Mäkitie O. Severe phenotype of APECED (APS1) increases risk for structural bone alterations. Frontiers in Endocrinology 202011 109. (https://doi. org/10.3389/fendo.2020.00109)

17 Tanner JM. Growth at Adolescence: With A General Consideration of the Effects of Hereditary and Environmental Factors upon Growth and Maturation from Birth to Maturity, 2nd ed. Oxford: Blackwell Publishing, 1962.

18 Saari A, Sankilampi U, Hannila ML, Kiviniemi V, Kesseli K \& Dunkel L. New Finnish growth references for children and adolescents aged 0 to 20 years: length/height-for-age, weight-forlength/height, and body mass index-for-age. Annals of Medicine 2011 43 235-248. (https://doi.org/10.3109/07853890.2010.515603)

19 Kantero R \& Widholm O. The age of menarche in Finnish girls in 1969. Acta Obstetricia et Gynecologica Scandinavica 197150 7-18. (https://doi.org/10.3109/00016347109155076)

20 Pennell LM, Galligan CL \& Fish EN. Sex affects immunity. Journal of Autoimmunity 201238 J282-J291. (https://doi.org/10.1016/j. jaut.2011.11.013)

21 Warren BD, Ahn SH, McGinnis LK, Grzesiak G, Su RW, Fazleabas AT, Christenson LK, Petroff BK \& Petroff MG. Autoimmune regulator is required in female mice for optimal embryonic development and implantation. Biology of Reproduction 2019100 1492-1504. (https:// doi.org/10.1093/biolre/ioz023)

22 Moreira-Filho CA, Bando SY, Bertonha FB, Ferreira LR, Vinhas CdF, Oliveira LHB, Zerbini MCN, Furlanetto G, Chaccur P \& CarneiroSampaio M. Minipuberty and sexual dimorphism in the infant human thymus. Scientific Reports 20188 13169. (https://doi. org/10.1038/s41598-018-31583-3)

23 Borchers J, Pukkala E, Mäkitie O \& Laakso S. Patients with APECED have increased early mortality due to endocrine causes, malignancies and infections. Journal of Clinical Endocrinology and Metabolism 2020 105 e2207-e2213. (https://doi.org/10.1210/clinem/dgaa140)

24 Ahonen P, Miettinen A \& Perheentupa J. Adrenal and steroidal cell antibodies in patients with autoimmune polyglandular disease type I and risk of adrenocortical and ovarian failure. Journal of Clinical Endocrinology and Metabolism 198764 494-500. (https://doi. org/10.1210/jcem-64-3-494)

25 Söderbergh A, Myhre AG, Ekwall O, Gebre-Medhin G, Hedstrand H, Landgren E, Miettinen A, Eskelin P, Halonen M, Tuomi T et al. Prevalence and clinical associations of 10 defined autoantibodies in autoimmune polyendocrine syndrome Type I. Journal of Clinical Endocrinology and Metabolism 200489 557-562. (https://doi. org/10.1210/jc.2003-030279)

Received 12 May 2020

Revised version received 3 August 2020

Accepted 13 August 2020 